\title{
Electron doping and superconductivity in the two-dimensional Hubbard model
}

\author{
D. Eichenberger and D. Baeriswyl \\ Department of Physics, University of Fribourg, CH-1700 Fribourg, Switzerland
}

\begin{abstract}
An elaborate variational wave function is used for studying superconductivity in the (repulsive) twodimensional Hubbard model, including both nearest- and next-nearest-neighbor hoppings. A marked asymmetry is found between the "localized" hole-doped region and the more itinerant electron-doped region. Superconductivity with $d$-wave symmetry turns out to be restricted to densities where the Fermi surface crosses the magnetic zone boundary. A concomitant peak in the magnetic structure factor at $(\pi, \pi)$ clearly points to a magnetic mechanism.
\end{abstract}

One of the central issues in the field of high-temperature superconductors has been-and for some researchers still is-the question whether pairing in the cuprates arises from purely repulsive interactions, as proposed by Anderson two decades ago. ${ }^{1}$ This question has been studied extensively in the framework of the two-dimensional (2D) (repulsive) Hubbard model (and the related $t-J$ model). Recent progress, both in dynamical mean-field theory ${ }^{2,3}$ and in variational calculations, ${ }^{4}$ has strengthened the case for the existence of a superconducting phase in the Hubbard model, with a ( $d$-wave) gap parameter reasonably close to the experimental values for intermediate interaction strengths ( $U$ of the order of the bandwidth). This conclusion has been challenged on the basis of Monte Carlo simulations, ${ }^{5}$ which we believe to be not conclusive, as discussed below.

Most previous studies of the Hubbard model have been restricted to nearest-neighbor hopping, where electron doping does not differ from hole doping. Here we show that the addition of second-neighbor hopping (which breaks the electron-hole symmetry) changes this behavior substantially. While the hole-doped side is "localized" and shows kineticenergy-driven superconductivity with a large condensation energy, the electron-doped side is itinerant with a potentialenergy-driven superconductivity and a small condensation energy.

The Hubbard Hamiltonian $\hat{H}=\hat{H}_{0}+U \hat{D}$ consists of a hopping term ("kinetic energy")

$$
\hat{H}_{0}=-\sum_{i, j, \sigma} t_{i j}\left(c_{i \sigma}^{\dagger} c_{j \sigma}+c_{j \sigma}^{\dagger} c_{i \sigma}\right)
$$

and an on-site repulsion $U \hat{D}$, where $\hat{D}=\sum_{i} n_{i \uparrow} n_{i \downarrow}$ is the number of doubly occupied sites, $n_{i \sigma}=c_{i \sigma}^{\dagger} c_{i \sigma}$, and the operator $c_{i \sigma}^{\dagger}\left(c_{i \sigma}\right)$ creates (annihilates) an electron at site $i$ with spin $\sigma$. We use the trial ground state

$$
|\Psi\rangle=e^{-h \hat{H}_{0} / t} e^{-g \hat{D}}\left|\Psi_{0}\right\rangle,
$$

where $g$ and $h$ are (real) variational parameters and $\left|\Psi_{0}\right\rangle$ is a BCS state with $d$-wave symmetry. The first term in Eq. (2) promotes delocalization and yields a substantial improvement with respect to the Gutzwiller wave function $(h=0)$. In fact, it has been demonstrated that ansatz (2) is very close to the exact ground state both for small $2 \mathrm{D}$ systems ${ }^{6}$ and for the solvable $1 / r$ chain. $^{7}$
In our previous study of the simple Hubbard model $\left(t_{i j}\right.$ $=t$ for nearest-neighbor sites and 0 otherwise),${ }^{8}$ we have found that wave function (2) exhibits $d$-wave pairing below a hole concentration of 0.18 . At first sight this result seems to contradict recent Monte Carlo simulations, ${ }^{5}$ where no signature for superconductivity was found, but a closer look shows that there is no discrepancy. In fact, three of the four hole densities considered in Ref. $5(0.18,0.22,0.28)$ are in a region where our variational ground state is also nonsuperconducting. For the remaining hole density of 0.09 the order parameter (for $U / t=6$, the lattice size is $8 \times 8$, as chosen in Ref. 5) is likely to be too small ${ }^{4}$ to be detected by the simulation.

The restriction to nearest-neighbor hopping leads to a (bare) Fermi surface which disagrees qualitatively with photoemission experiments on layered cuprates. ${ }^{9}$ Therefore we consider from now on the more realistic case of both nearest$(t)$ and next-nearest-neighbor hoppings $\left(t^{\prime}\right)$ with canonical parameters $U=8 t$ and $t^{\prime}=-0.3 t$. The bare single-particle spectrum,

$$
\epsilon_{\vec{k}}=-2 t\left(\cos k_{x}+\cos k_{y}\right)-4 t^{\prime} \cos k_{x} \cos k_{y},
$$

leads to the Fermi surfaces of Fig. 1. The innermost line corresponds to the Van Hove filling where the Fermi surface passes through the saddle points at $(\pi, 0)$ and $(0, \pi)$. These crossings between the Fermi surface and the magnetic zone boundary (the "hot spots") move inward as the density $n$ is increased and finally merge (outermost line). Hot spots are

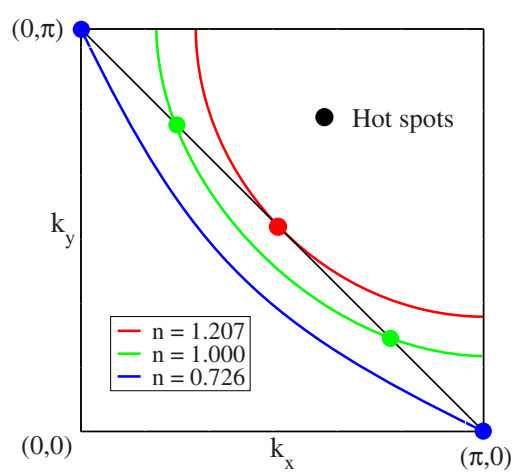

FIG. 1. (Color online) Fermi surface for three different electron densities. 
TABLE I. Chemical potential, parameters $g$ and $h$, and energy per site for hole doping $(n<1)$ and an $8 \times 8$ lattice.

\begin{tabular}{ccccc}
\hline \hline$n$ & $\mu$ & $g$ & $h$ & $E / t$ \\
\hline 0.7500 & $-0.9921(1)$ & $4.2(1)$ & $0.113(2)$ & $-0.858(1)$ \\
0.7800 & $-0.9612(3)$ & $4.0(1)$ & $0.112(2)$ & $-0.829(1)$ \\
0.8125 & $-0.9107(3)$ & $3.9(1)$ & $0.111(2)$ & $-0.795(1)$ \\
0.8400 & $-0.788(1)$ & $3.7(1)$ & $0.111(2)$ & $-0.763(1)$ \\
0.9000 & $-0.728(1)$ & $3.8(1)$ & $0.111(2)$ & $-0.676(1)$ \\
0.9500 & $-0.603(1)$ & $4.0(1)$ & $0.114(2)$ & $-0.591(1)$ \\
\hline \hline
\end{tabular}

restricted to $0.726<n<1.206$. Our results, to be discussed below, indicate that superconductivity occurs only in this range.

The BCS state $\left|\Psi_{0}\right\rangle$ in Eq. (2) introduces, in addition to $g$ and $h$, two other parameters: the amplitude $\Delta$ of the superconducting gap function and the "chemical potential" $\mu$. To compute the variational energy, the exponent of the operator $e^{-g \hat{D}}$ is first decoupled by applying a discrete HubbardStratonovich transformation, ${ }^{10}$ which introduces an Ising spin at each site. All operators are then quadratic in creation and annihilation operators, and therefore the fermionic degrees of freedom can be integrated out. The remaining sum over Ising spin configurations is performed by a Monte Carlo simulation. In order to avoid the minus sign problem, calculations are carried out in the grand canonical ensemble with an average density fixed by $\mu$, which is therefore not a variational parameter. To reduce the statistical error, the optimization procedure is based on the method proposed by Ceperley et $a .^{11}$ and Umrigar et al. ${ }^{12}$ We have used periodicantiperiodic boundary conditions.

The minimization of the energy $E$ for fixed average densities yields the results of Tables I and II for hole and electron dopings, respectively. We notice that the parameter $g$, which controls the crossover between itinerant (small $g$ ) and localized (large $g$ ) many-particle states, remains large for hole doping but decreases rapidly for electron doping. Therefore, while the hole-doped region $0.75<n<0.95$ is a localized doped Mott insulator, the electron-doped part $1.05<n$ $<1.2$ rapidly undergoes a crossover to an itinerant regime as $n$ increases. (We estimate a crossover parameter $g_{\text {cr }} \approx 3$.) This striking difference may root in the bare single-particle density of states at the Fermi energy, which is much larger for hole than for electron doping. We notice that our wave

TABLE II. Chemical potential, parameters $g$ and $h$, and energy per site for electron doping $(n>1)$ and an $8 \times 8$ lattice.

\begin{tabular}{ccccc}
\hline \hline$n$ & $\mu$ & $g$ & $h$ & $E / t$ \\
\hline 1.0500 & $0.7666(1)$ & $3.6(1)$ & $0.109(2)$ & $-0.222(1)$ \\
1.0800 & $0.6440(1)$ & $3.4(1)$ & $0.106(2)$ & $-0.069(1)$ \\
1.1000 & $0.5488(1)$ & $3.2(1)$ & $0.104(2)$ & $0.040(1)$ \\
1.1300 & $0.4380(1)$ & $3.0(1)$ & $0.100(2)$ & $0.206(1)$ \\
1.1600 & $0.3870(1)$ & $2.9(2)$ & $0.096(3)$ & $0.374(1)$ \\
1.2000 & $-0.2996(1)$ & $2.6(2)$ & $0.091(3)$ & $0.608(1)$ \\
\hline \hline
\end{tabular}

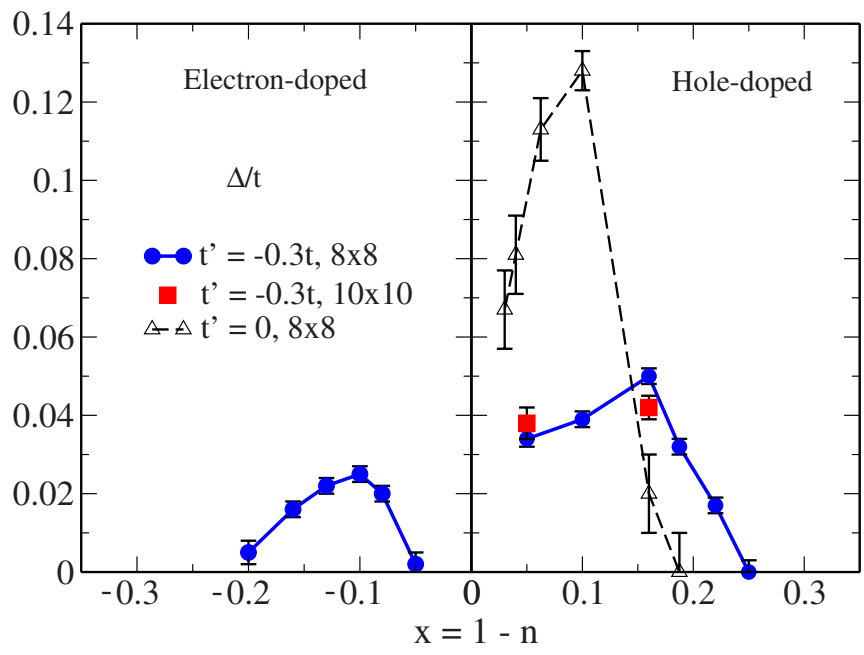

FIG. 2. (Color online) Optimized gap parameter as a function of doping for two different lattice sizes. For comparison, the result for $t^{\prime}=0$ is also shown (from Ref. 8).

function is excellent for the itinerant regime and somewhat less reliable for the localized regime. ${ }^{7}$

Figures 2 and 3 show, respectively, the gap parameter $\Delta$ and the superconducting order parameter $\Phi=\left|\left\langle c_{i \uparrow}^{\dagger} c_{j_{i} \downarrow}^{\dagger}\right\rangle\right|$ as functions of doping concentration $x=1-n$. The corresponding results for $t^{\prime}=0$ (Ref. 8) are completely electron-hole symmetric and are reproduced only in the right panels. On the hole-doped side superconductivity exists for $0<x$ $<0.25$ ( $\Delta$ remains finite, but $\Phi$ vanishes for $x \rightarrow 0$ ), i.e., in a larger region than for $t^{\prime}=0$. In contrast, on the electrondoped side the superconducting region is reduced to -0.2 $<x<-0.05$. Thus we find indeed that superconductivity is restricted to densities where the (bare) Fermi surface passes through hot spots (see Fig. 1). Remarkably, for electron doping the gap is suppressed very close to half filling even in the absence of competing antiferromagnetic long-range order. The marked difference between electron and hole dopings is confirmed by the condensation energy $E_{\text {cond }}=E(0)-E(\Delta)$, which is 1 order of magnitude smaller for electron doping than for hole doping, as depicted in Fig. 4.

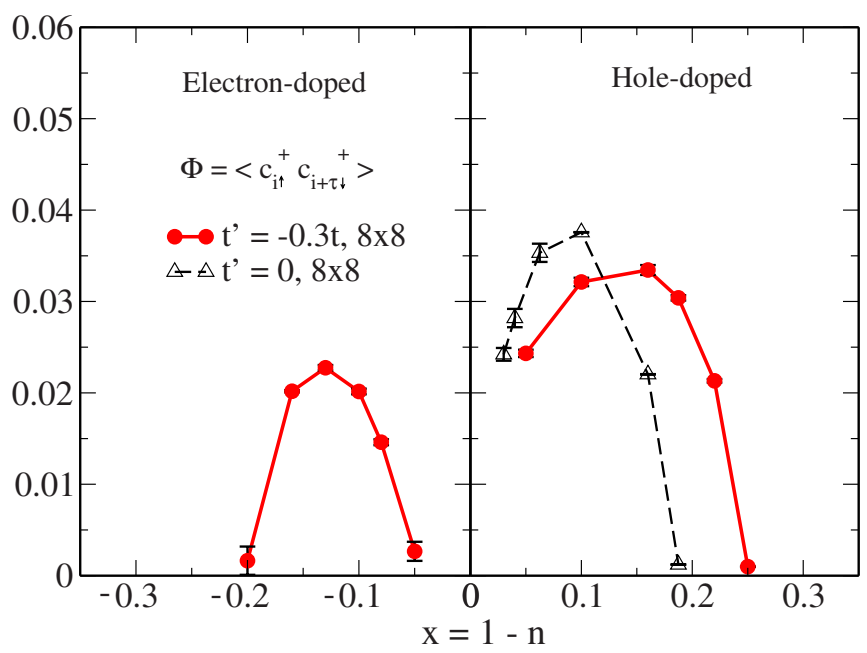

FIG. 3. (Color online) Superconducting order parameter as a function of doping for an $8 \times 8$ lattice. 


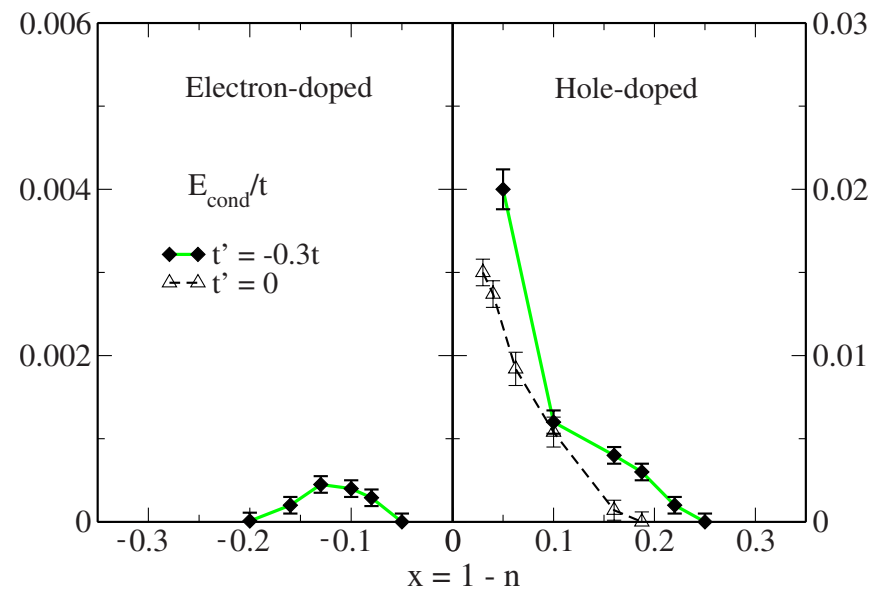

FIG. 4. (Color online) Condensation energy per site for an $8 \times 8$ lattice.

Figure 5 shows the kinetic, potential, and total energies as functions of the gap parameter for the two densities $n$ $=0.78$ and 1.16. For hole doping the energy gain is clearly due to a decrease in kinetic energy, while for electron doping the decrease in potential energy gives the main contribution to the condensation energy. The same behavior has been consistently obtained for other densities. The quantity

$$
W=-2 \sum_{\vec{k}} \frac{\partial^{2} \epsilon_{\vec{k}}}{\partial k_{x}^{2}}\left\langle n_{\vec{k}}\right\rangle,
$$

also plotted in Fig. 5, is - up to a minus sign-proportional to the integrated optical conductivity originating from intraband transitions. ${ }^{13}$ For the simple Hubbard model $\left(t^{\prime}=0\right) \mathrm{W}$ is equal to the kinetic energy, but for $t^{\prime} \neq 0$ the two quantities differ. For hole doping $W$ has a pronounced minimum in the region of the optimal gap, corresponding to an increase in oscillator strength, while for electron doping $W$ increases monotonically with $\Delta$, akin to the BCS behavior where the

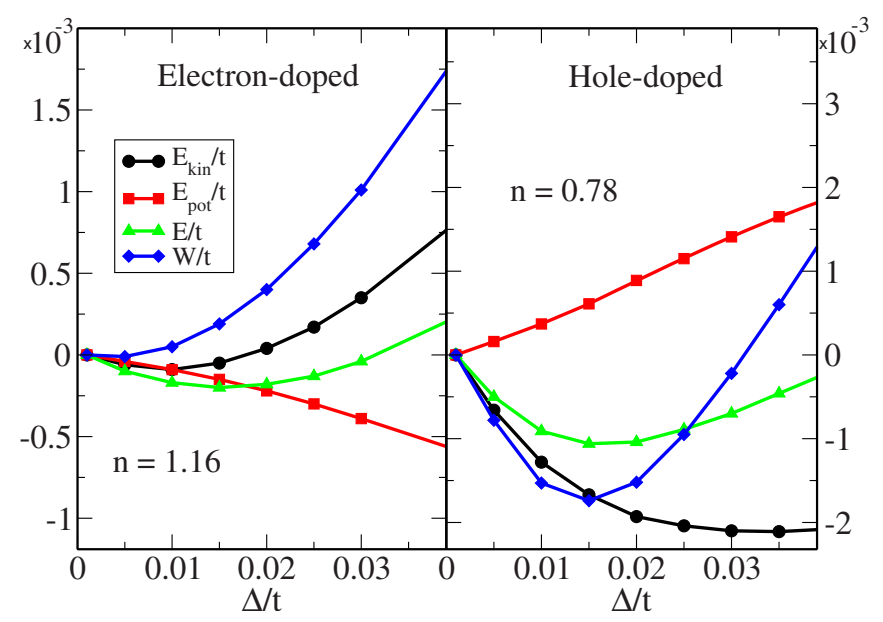

FIG. 5. (Color online) Changes in kinetic, potential, and total energies as well as in the quantity $W$ as functions of the gap parameter for an $8 \times 8$ lattice. ( $-W$ is proportional to the oscillator strength for intraband transitions.) The relative uncertainties are smaller than the symbol sizes.

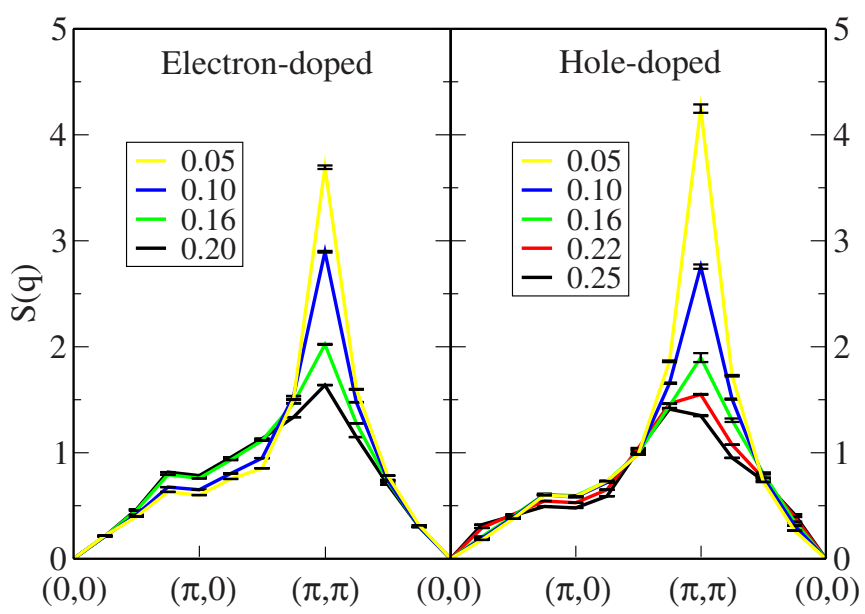

FIG. 6. (Color online) Magnetic structure factor as a function of the momentum, for various densities above and below half filling, for an $8 \times 8$ lattice.

oscillator strength is reduced at the onset of superconductivity.

We attribute this asymmetry between hole and electron dopings to the different values of the correlation parameter $g$ (see Tables I and II), which puts the hole-doped system into the localized regime, while the electron-doped system is more itinerant. To make the point clear we consider the simple Hubbard model $\left(t^{\prime}=0\right)$ both in the small $U$ (itinerant) and large $U$ (localized) limits. In the small $U$ limit superconductivity is produced by the Kohn-Luttinger mechanism, ${ }^{14}$ where the condensation energy is expected to increase as a function of $U / t$, whereas in the large $U$ limit the condensation energy arises from magnetic exchange and thus is likely to increase with $t / U$. The change in kinetic energy is then obtained through the Hellman-Feynman theorem,

$$
E_{\mathrm{kin}}(\Delta)-E_{\mathrm{kin}}(0)=-t \frac{\partial}{\partial t} E_{\mathrm{cond}},
$$

which is positive in the small $U$ limit and negative in the large $U$ limit.

Additional information can be gained from the magnetic structure factor

$$
S(\vec{q})=\frac{1}{N} \sum_{i, j} e^{i \vec{q} \cdot\left(\vec{R}_{i}-\vec{R}_{j}\right)}\left\langle\left(n_{i \uparrow}-n_{i \downarrow}\right)\left(n_{j \uparrow}-n_{j \downarrow}\right)\right\rangle,
$$

displayed in Fig. 6. $S(\vec{q})$ exhibits a clear maximum at $(\pi, \pi)$, which is largest close to half filling and decreases as doping increases. There is very little difference between electron and hole dopings, presumably because in the large $U$ limit spin correlations depend mostly through the exchange constants $J=4 t^{2} / U$ and $J^{\prime}=4 t^{\prime 2} / U$ on the microscopic parameters and therefore are essentially independent of the sign of $t^{\prime}$.

We have seen above that superconductivity is restricted to the region where two points of the Fermi surface can be connected (at least approximately) by the antiferromagnetic wave vector $(\pi, \pi)$. This together with the strong peak of $S(\vec{q})$ for $\vec{q}=(\pi, \pi)$ supports the point of view that superconductivity in the two-dimensional Hubbard model is due to a 
magnetic mechanism. This conclusion is now widely accepted, but the question whether the mechanism is of the resonating valence bond (RVB) type ${ }^{1}$ or arises from the exchange of spin fluctuations ${ }^{15}$ is presently under debate. ${ }^{16,17}$ We cannot solve this problem on the basis of our variational calculations, but the distinction between localized and itinerant regimes can give additional useful insight. According to our results the former is appropriate for the hole-doped region, where superconductivity is associated with a decrease in kinetic energy; the latter regime is found for electron doping, where superconductivity arises from a more conventional gain in potential energy.

The results described above compare surprisingly well with experiments on cuprates, better than our earlier calculations ${ }^{8}$ where only nearest-neighbor hopping has been taken into account. This concerns the phase diagram, in particular, for electron-doped materials for which a recent systematic study ${ }^{18}$ gives a dome shape (for $T_{c}$ ) strikingly similar to our Fig. 2. Photoemission data give values of the superconducting gap $\Delta$ in the range $10-20 \mathrm{meV}$ for hole-doped compounds $\left[\mathrm{La}_{2-x} \mathrm{Sr}_{x} \mathrm{CuO}_{4+y}\right.$ (LSCO) (Ref. 19) or Bi2201 (Ref. 9)] and $\sim 5 \mathrm{meV}$ for an electron-doped compound [NdCeCO (Ref. 20)]. Choosing $t=300 \mathrm{meV}$ (neutron data),

${ }^{1}$ P. W. Anderson, Science 235, 1196 (1987).

${ }^{2}$ T. A. Maier, M. Jarrell, and D. J. Scalapino, Phys. Rev. B 74, 094513 (2006).

${ }^{3}$ K. Haule and G. Kotliar, Phys. Rev. B 76, 104509 (2007).

${ }^{4}$ H. Yokoyama, Y. Tanaka, M. Ogata, and H. Tsuchira, J. Phys. Soc. Jpn. 73, 1119 (2004).

${ }^{5}$ T. Aimi and M. Imada, J. Phys. Soc. Jpn. 76, 113708 (2007).

${ }^{6}$ H. Otsuka, J. Phys. Soc. Jpn. 61, 1645 (1992).

${ }^{7}$ M. Dzierzawa, D. Baeriswyl, and M. Di Stasio, Phys. Rev. B 51, 1993 (1995).

${ }^{8}$ D. Eichenberger and D. Baeriswyl, Phys. Rev. B 76, 180504(R) (2007).

${ }^{9}$ A. Damascelli, Z. Hussain, and Z.-X. Shen, Rev. Mod. Phys. 75, 473 (2003)

${ }^{10}$ J. E. Hirsch, Phys. Rev. B 28, 4059 (1983).

${ }^{11}$ D. Ceperley, G. V. Chester, and K. H. Kalos, Phys. Rev. B 16, 3081 (1977).

${ }^{12}$ C. J. Umrigar, K. G. Wilson, and J. W. Wilkins, Phys. Rev. Lett. our maximum gap parameters are 15 and $7 \mathrm{meV}$ for hole and electron dopings, respectively. An increase in oscillator strength, predicted by our calculations for hole doping, has been reported on the basis of optical spectroscopy for an underdoped sample, ${ }^{21}$ but the situation is less clear on the overdoped side. We are not aware of corresponding measurements on electron-doped materials.

In conclusion, our variational search for $d$-wave superconductivity in the two-dimensional Hubbard model gives a differentiated picture, namely, a large (moderate) correlation parameter for hole (electron) doping, a gain in kinetic (potential) energy due to pairing, and a large (small) condensation energy. Our wave function is expected to be better suited for describing the electron-doped region, which is less localized than the hole-doped region. A more elaborate study is needed to improve our understanding of the pseudogap phase observed in the cuprates for weak hole doping.

We are grateful for financial support from the Swiss National Science Foundation through the National Center of Competence in Research "Materials with Novel Electronic Properties-MaNEP." The computations have been partially performed on the Pléiades cluster of the EPFL.

60, 1719 (1988).

${ }^{13}$ D. Baeriswyl and L. Degiorgi, Strong Interactions in Low Dimensions: Physics and Chemistry of Materials with LowDimensional Structures (Kluwer, Dordrecht, 2004), Vol. 25.

${ }^{14}$ W. Kohn and J. M. Luttinger, Phys. Rev. Lett. 15, 524 (1965).

${ }^{15}$ D. Scalapino, J. Low Temp. Phys. 117, 179 (1999).

${ }^{16}$ P. W. Anderson, Science 316, 1705 (2007).

${ }^{17}$ T. A. Maier, D. Poilblanc, and D. J. Scalapino, Phys. Rev. Lett. 100, 237001 (2008).

${ }^{18}$ Y. Krockenberger, J. Kurian, A. Winkler, A. Tsukada, M. Naito, and L. Alff, Phys. Rev. B 77, 060505(R) (2008).

${ }^{19}$ K. Yamada, S. Wakimoto, G. Shirane, C. H. Lee, M. A. Kastner, S. Hosoya, M. Greven, Y. Endoh, and R. J. Birgeneau, Phys. Rev. Lett. 75, 1626 (1995).

${ }^{20}$ T. Sato, T. Kamiyama, T. Takahashi, K. Kurahashi, and K. Yamada, Science 291, 1517 (2001).

${ }^{21}$ F. Carbone et al., Phys. Rev. B 74, 064510 (2006). 